\title{
Star formation around three co-moving HAeBe stars in the Cepheus Flare (Corrigendum)
}

\author{
P. Saha ${ }^{1,2}$, G. Maheswar ${ }^{1}$, B. Mathew ${ }^{3}$, and U. S. Kamath ${ }^{1}$ \\ 1 Indian Institute of Astrophysics (IIA), Sarjapur Road, Koramangala, Bangalore 560034, India \\ e-mail: s.piyali16@gmail.com \\ 2 Pt. Ravishankar Shukla University, Amanaka G.E. Road, Raipur, Chhatisgarh 492010, India \\ 3 Department of Physics and Electronics, CHRIST (Deemed to be University), Bangalore 560029, India
}

A\&A 653, A142 (2021), https://doi.org/10.1051/0004-6361/202039646

Key words. parallaxes - proper motions - stars: formation - stars: pre-main sequence - ISM: clouds - errata, addenda

An error occurred during the production process. In Sect. 4 (Summary and conclusion), two paragraphs were misplaced. The first paragraph should be "We studied the kinematics ...," whereas paragraph "More rigorous study ..." should be placed separately, after the enumeration. 